\title{
COMENTARIO
}

\section{Incendios en bosques de Araucaria araucana y consideraciones ecológicas al madereo de aprovechamiento en áreas recientemente quemadas}

\author{
Wildfire in Araucaria araucana forests and ecological considerations about salvage \\ logging in areas recently burned
}

\author{
MAURO E. GONZÁLEZ ${ }^{1, *} \&$ THOMAS T. VEBLEN ${ }^{2}$ \\ ${ }^{1}$ Instituto de Silvicultura, Núcleo Científico FORECOS, Universidad Austral de Chile, Casilla 567, Valdivia, Chile \\ ${ }^{2}$ Department of Geography, University of Colorado, Boulder, Colorado 80302, USA \\ *e-mail para correspondencia: maurogonzalez@uach.cl
}

\begin{abstract}
RESUMEN
El fuego es el tipo de disturbio más importante en conformar el paisaje forestal de Araucaria araucana en la zona centro-sur de Chile y Argentina. En la Región de la Araucanía el clima y el ser humano han influenciado el régimen de fuego durante al menos los últimos mil años. Los incendios catastróficos ocurridos recientemente (año 2002) que afectaron los bosques de Araucaria atrajeron la atención pública, política y científica generando una importante necesidad de entender las causas y efectos de tales eventos en los ecosistemas forestales. Estos grandes incendios produjeron una abundante cantidad de material muerto en pie y en el suelo, promoviendo el desarrollo de otros atributos estructurales conocidos como legados biológicos que tienen un rol crítico en la recuperación de los ecosistemas luego de este tipo de eventos. El madereo de aprovechamiento ("salvage logging") es considerado un factor negativo en la recuperación de áreas afectadas significando tanto una pérdida o menoscabo de la capacidad de regeneración como un deterioro en otros procesos ecológicos clave. Incendios catastróficos de alta severidad, ya sea antrópicos o naturales, crean presiones tanto para reemplazar el bosque nativo por plantaciones de especies de crecimiento rápido o llevar a cabo operación de madereo de aprovechamiento. Las decisiones de manejo deben estar basadas en un mejor entendimiento de la ocurrencia pasada de incendios y su rol en modelar los presentes ecosistemas de Araucaria. Particularmente, en el contexto del excepcional valor ecológico y cultural de estos ecosistemas, las agencias y profesionales del manejo forestal deberían considerar políticas que efectivamente aseguren y sean consistentes con la restauración de procesos ecológicos y atributos clave en los ecosistemas de Araucaria.
\end{abstract}

Palabras clave: Araucaria araucana, madereo de aprovechamiento, incendios, historia de fuego.

\begin{abstract}
Fire is the most important kind of disturbance shaping the Araucaria araucana forest landscapes of southcentral Chile and Argentina. In the Araucarian region, climate and humans have influenced historical fire regimes over many centuries. In the summer of 2002 widespread, severe fires burned in Chilean Araucaria forests and became the center of much interest among politicians, scientists and the general public. The 2002 fires focused attention on the importance of understanding the causes and effects of such severe events in the forest ecosystems. Major wildfires generate large quantities of dead and downed trees, and promote the development of other structural attributes known as biological legacies that play critical roles in ecosystem recovery after such events. The potential for severe fire, either of natural or human origin, create pressures to either replace native forests with plantations of fast-growing exotic trees or to conduct salvage logging. Salvage harvesting can impede or alter the trajectory of recovery of affected areas, and it potentially may decrease forest regeneration and have detrimental impacts on key ecological processes. Forest policy and management decisions should be informed by a better understanding of the past occurrence of fire and its role in shaping the modern Araucaria forest ecosystem. Particularly, in the context of the exceptional ecological and cultural value of these ecosystems, managers should consider policies that effectively secure and are consistent with the restoration of the key attributes and processes of Araucaria forest ecosystems.
\end{abstract}

Key words: Araucaria araucana, salvage logging, wildfire, fire history. 


\section{INTRODUCCIÓN}

Durante la temporada estival 2001-2002 presenciamos uno de los mayores incendios de los últimos 50 años en la Región de la Araucanía. En la cordillera andina de las provincias de Malleco y Cautín, cerca de 20.000 ha de bosques fueron consumidas por el fuego de las cuales un $30 \%$ correspondió a bosques mixtos de Araucaria-Nothofagus, incluyendo tanto áreas silvestres protegidas como privadas (CONAF 2002). Estos grandes incendios provocaron una gran conmoción pública y política considerando la importancia ecológica y cultural de la especie Araucaria araucana (Echeverría 2002). Además, contraria a la percepción generalizada de que los incendios son derivados exclusivamente de la actividad humana $-\mathrm{y}$ por ende vistos como un fenómeno ajeno y negativo a los ecosistemas(González 2005), de gran interés y sorpresa resultó conocer que los principales focos de estos incendios catastróficos fueron causados por rayos, derivados de tormentas eléctricas secas durante el verano (CONAF 2002). Esta nota tiene por finalidad clarificar el rol preponderante del fuego en estos ecosistemas boscosos, y discutir los potenciales riesgos del madereo ("salvage harvesting") en la recuperación de las áreas recientemente quemadas.

El fuego en los bosques de Araucaria araucana: un proceso ecológico clave

La presencia del fuego como agente modelador del paisaje en la región andina sudamericana se señala al menos para los últimos 40.000 años (Heusser 1983, 1994). Araucaria araucana se ubica en una región volcánica muy activa que sin duda ha dirigido las adaptaciones de esta conífera como de otras especies asociadas (e.g., Nothofagus) a disturbios por fuego (Montaldo 1974, Veblen et al. 1995, Veblen et al. 1996, Bekessy et al. 2004, González et al. 2005). Su corteza gruesa (> $10 \mathrm{~cm}$ en espesor), la poda natural de las ramas bajas formando el parasol característico de los individuos adultos, y su capacidad de rebrote vegetativo, señalan la capacidad de esta especie para contrarrestar y responder a los incendios tanto superficiales como catastróficos.
El fuego juega un importante papel en la estructura y dinámica de los bosques de Araucaria-Nothofagus, a escala tanto de rodal como de paisaje. El régimen de fuego en estos ecosistemas es caracterizado por una combinación de incendios superficiales relativamente frecuentes de baja intensidad asociados con una menor mortalidad, e incendios catastróficos infrecuentes que resultan en una alta mortalidad del dosel dominante (González et al. 2005). Tanto individuos adultos de Araucaria y Nothofagus como rodales completos dominados por estas especies -asociados a quebradas y cursos de agua- son capaces de resistir y sobrevivir incendios de gran severidad (Peñaloza 2006), representando el legado y fuente de propágulos para el restablecimiento de la nueva cohorte de individuos. La respuesta postfuego en rodales totalmente destruidos resulta típicamente en la rápida y masiva colonización de especies de Nothofagus $-N$. pumilio, $N$. dombeyi o $N$. nervosa según altitud, exposición y ubicación geográfica-, junto a un más lento y disperso establecimiento de individuos de Araucaria. Así, en este proceso un importante papel juega el legado de árboles sobrevivientes tanto de individuos de Araucaria y Nothofagus (González 2002).

La historia del fuego en bosques de Araucaria araucana refleja tanto la influencia climática como de la actividad humana, incluidos los pueblos originarios y colonos de origen italiano, suizo, alemán, francés y español principalmente, además de chilenos, establecidos en la Región de la Araucanía a fines del siglo XIX (Pereira 1965). Los incendios en la región andina centro-sur de Chile y norpatagónica de Argentina, han sido asociados importantemente con eventos ENOS (El Niño Oscilación del Sur) y otros patrones de circulación atmosférica de gran escala (e.g., Anticiclón del Pacífico, Kitzberger et al. 1997, Kitzberger \& Veblen 1997, Veblen et al. 1999, González \& Veblen 2006). Por ejemplo, una mayor incidencia de incendios ha sido asociada con un mayor desarrollo y una ubicación más meridional del Anticiclón del Pacífico, el cual bloquea los frentes de precipitación hacia el continente, provocando primaveras y veranos más secos. A su vez esta amplificación del anticiclón permite la incursión en la época 
estival de frentes subtropicales provenientes del noreste argentino. Estas masas de aire húmedo generan tormentas eléctricas y rayos, que coincidentes con un verano seco pueden ser la causa natural de incendios de grandes proporciones en la región (Kitzberger et al. 1997). Además, incendios de gran magnitud han sido relacionados con la variación climática interanual provocada por eventos ENOS. Particularmente, la transición de la Niña (periodo relativamente seco) al Niño (periodo más cálido), y también veranos más cálidos que siguen típicamente un evento $\mathrm{El}$ Niño (asociado a un invierno lluvioso; Montecinos \& Aceituno 2003) caracterizan las condiciones típicas de años con mayor número y extensión de los incendios en esta región.

La influencia humana en la Región de la Araucanía también ha jugado un papel importante en el mosaico de los actuales bosques de Araucaria, especialmente como resultado de la colonización eurochilena a partir de la década de 1880 . Desde este periodo los bosques de Araucaria-Nothofagus distribuidos tanto en Chile como Argentina comienzan a ser intensamente destruidos por incendios intencionales (Baquedano 1914, Elizalde 1958, Lara et al. 1996, Donoso \& Lara 1996). El fuego fue utilizado como la herramienta predilecta para abrir y mantener áreas de pastoreo. Además, muchas veces el fuego fue usado para facilitar las labores de madereo o una vez terminado este, para definitivamente habilitar el bosque a fines agrícolas o ganaderos. Por ejemplo, en bosques de Araucaria-Nothofagus ubicados en las faldas del Volcán Lanín en el Parque Nacional Villarrica la frecuencia de incendios durante el siglo XX al menos dobló aquella de los siglos precedentes (siglos XVIII y XIX, González et al. 2005, Fig. 1). Este notable aumento en la frecuencia de incendios coincidió con el arribo de colonos euro-chilenos a la región luego de 1883, fecha que marca la definitiva incorporación de la Región de la Araucanía al territorio nacional. Similarmente, en el Parque Nacional Tolhuaca una mayor ocurrencia de incendios se aprecia notablemente desde 1900 hasta alrededor de 1960. Posterior a 1960 la menor ocurrencia de incendios coincidiría con la mayor eficacia en el control y prevención de incendios luego de la creación de este parque. En este periodo, entre 1960 y 2002, el único incendio de gran magnitud e impacto en el paisaje -ya que el incendio de 1987 fue de carácter local- fue el originado naturalmente en febrero del año 2002 por una tormenta eléctrica (rayos).

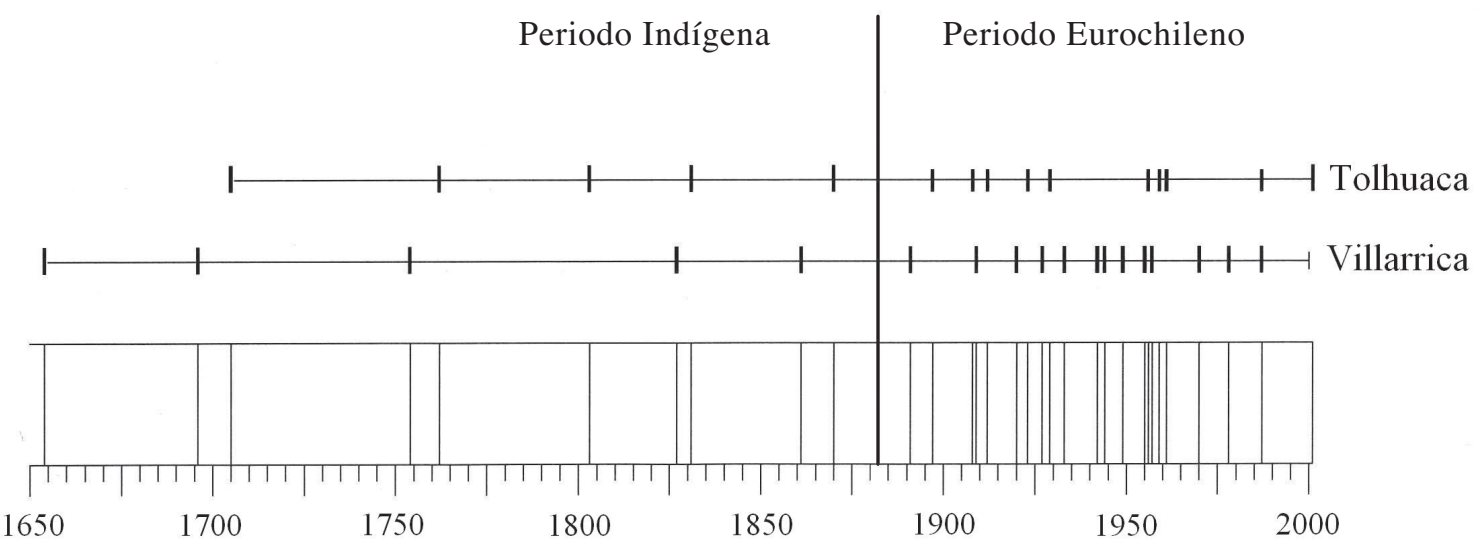

Fig. 1: Resumen de registros de incendios en el Parque Nacional Tolhuaca y Villarrica. Fechas de incendios antes de 1650 no se incluyen. Las fechas de incendio son indicadas por líneas cortas verticales. Las líneas verticales que se extienden al eje X indican el resumen de todas las fechas de incendio para ambas áreas de estudio.

Composite fire-scar records for Tolhuaca and Villarrica National Parks. Fire dates before 1650 are not included. Dates of fire scars are indicated by short vertical lines. Vertical lines extending to the $\mathrm{x}$-axis indicate dates of fires in both study areas. 
Incendios catastróficos, legados biológicos, y efectos del madereo de salvamento

Los disturbios naturales han sido reconocidos como los principales factores de cambio en la vegetación y considerados no como eventos de rara ocurrencia, y por lo tanto, ajenos al sistema, sino como parte inherente de ellos (Spies \& Turner 1999). Actualmente y sustentados en los nuevos paradigmas que consideran la naturaleza dinámica y de no equilibrio de los sistemas ecológicos (Pickett \& White 1985, Glenn-Lewin et al. 1992), los disturbios naturales son considerados como procesos ecosistémicos clave en vez de desastres ecológicos que requieren de la ayuda y reparación humana (Lindenmayer et al. 2004).

Disturbios de gran escala, como los incendios catastróficos ocurridos en la Región de la Araucanía en el verano de 2002, generan una abundante variedad de legados biológicos que corresponden a organismos o estructuras derivadas orgánicamente (e.g., troncos caídos, árboles muertos en pie, árboles sobrevivientes, parches de vegetación de sotobosque) que ofrecen un amplio rango de funciones en la recuperación de la comunidad forestal (Franklin et al. 2000, Lindenmayer \& Franklin 2002 y sus citas). La capacidad de respuesta de la vegetación luego de severos disturbios (e.g., fuego) está determinada por factores y procesos tales como supervivencia de árboles, respuesta de la regeneración avanzada, nuevo establecimiento vía semillas y rebrotes vegetativos, sustrato y fuente de semillas, clima, entre otros. El tipo y severidad de un disturbio natural afecta directamente la cantidad, tipos y patrones espaciales de los legados biológicos que permanecen en el área afectada y estos afectan o controlan importantemente la dirección sucesional y los procesos de recuperación postdisturbio (Turner et al. 1998, Franklin et al. 2000, Tabla 1). La tala rasa -así como el madereo de rescate- es un tipo de alteración que no tiene análogo al tipo y características derivadas de los disturbios naturales $\mathrm{y}$, por lo tanto, la remoción de gran parte del material leñoso puede cambiar sustantivamente el funcionamiento y respuesta del ecosistema. En contraste, alteraciones naturales como incendios o caídas masivas por viento resultan generalmente en condiciones -estructurales, funcionales y medioambientales- que favorecen el reclutamiento y recuperación de los ecosistemas afectados.

Estudios recientemente iniciados en el Parque Nacional Tolhuaca, posterior al incendio de febrero del 2002, muestran el rol fundamental de los árboles muertos en pie y árboles sobrevivientes de Araucaria y Nothofagus -y de otras especies arbustivascomo fuente de propágulos y protección para el establecimiento de las especies que reconstruirán la comunidad forestal (M.E. González, resultados no publicados). En las áreas afectadas por el incendio el tipo y variedad de los legados biológicos estuvo determinado importantemente por su severidad. Una alta severidad resultó en el consumo por el fuego de todo el combustible de tamaño fino y mediano (especies de sotobosque y individuos $<5 \mathrm{~cm}$ DAP) y una completa mortalidad del componente arbóreo

TABLA 1

Diferencias en tipo y cantidad de legado biológico luego de distintos tipos de disturbios en ecosistemas forestales (modificado de Franklin et al. 2000)

Differences in the kind and quantities of biological legacies following different types of forest disturbances (modified from Franklin et al. 2000)

\begin{tabular}{lccc}
\hline Tipo de legado & & Tipo de disturbio & \\
\cline { 2 - 4 } & Fuego & Viento & Tala rasa \\
\hline Árboles muertos en pie & Abundante & Poco & Ninguno \\
Troncos & Común & Abundante & Poco o ninguno \\
Disturbio del suelo & Bajo & Parchado & Alto \\
Plantas de sotobosque & Común & Abundante & Poco \\
Nutrientes del suelo & Alto en N y P & Sin cambios & Alto en N y P \\
\hline
\end{tabular}


(Fig. 2A y 2B). En cambio en áreas afectadas con una menor severidad, una importante proporción tanto de especies de sotobosque y arbóreas fueran capaces de sobrevivir (Fig. 2C y 2D). Luego de tres periodos vegetativos postincendio, las áreas afectadas han sido colonizadas de manera relativamente rápida a través de nuevos establecimientos vía semillas -principalmente en el caso de los rodales afectados con una mayor severidad-y rebrotes vegetativos de especies cuyos rizomas, raíces, bulbos y otras estructuras subterráneas,

(A)

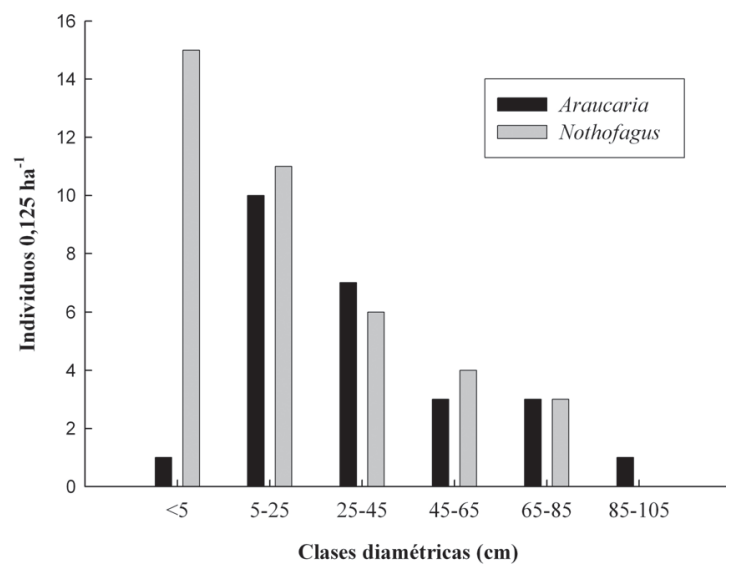

(C)

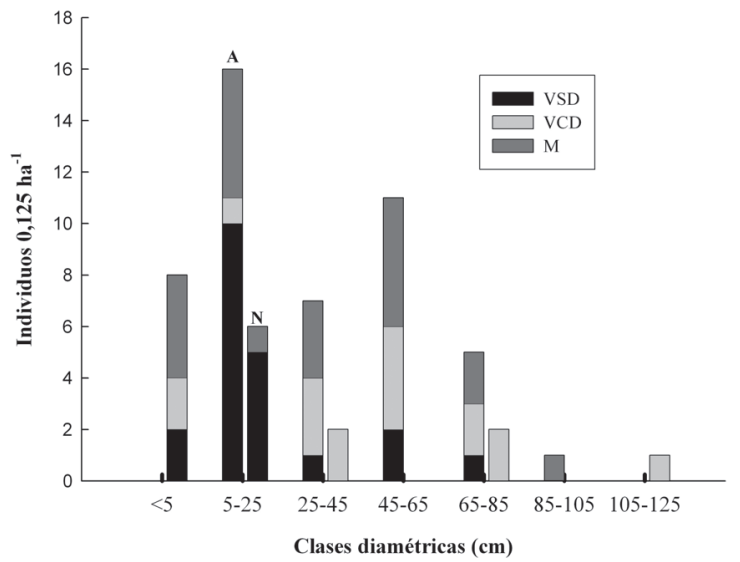

lograron sobrevivir al incendio (Tabla 2). En ambas áreas, Chusquea culeou fue la especie que colonizó más rápida y densamente a través de su sistema de rizomas. Es interesante notar para el caso de los rodales afectados más severamente, el rápido establecimiento de especies alóctonas, probablemente asociadas al ingreso de ganado al Parque. Esto sugiere que la actividad de madereo en bosques recientemente quemados sería una vía directa de invasión de malezas con consecuencias inciertas en la comunidad.
(B)

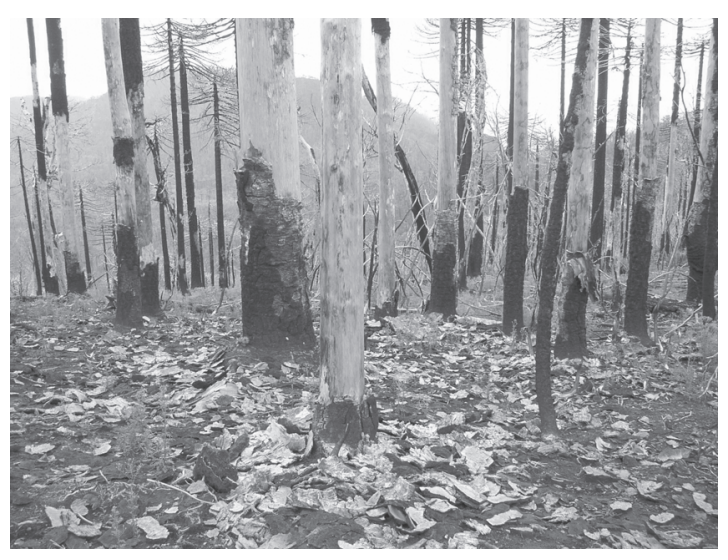

(D)

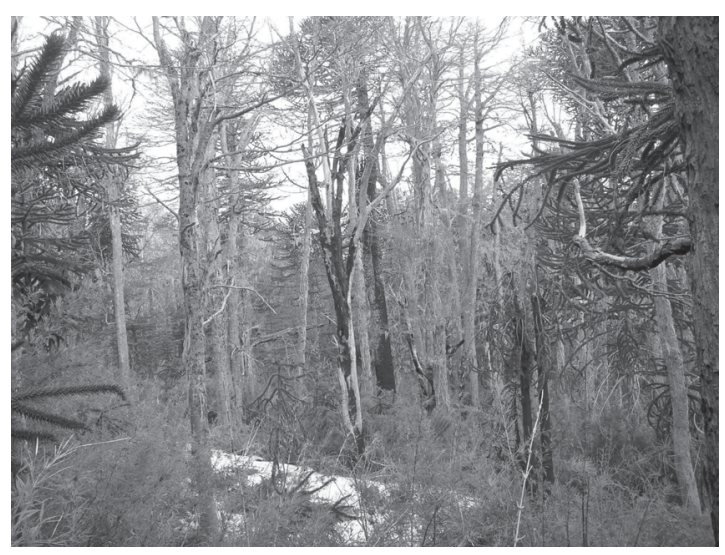

Fig. 2: (A) Estructura diamétrica de un bosque adulto de Araucaria-Nothofagus sin sobrevivientes luego de un año del incendio (2002), (B) aspecto del bosque luego del incendio de alta severidad, (C) Estructura diamétrica de un bosque adulto de Araucaria-Nothofagus que incluye árboles vivos sin daño de copa (VSD), árboles vivos con daño parcial a la copa (VCD), y árboles muertos (M) luego del incendio ( $\mathrm{A}=$ Araucaria $; \mathrm{N}=$ Nothofagus $)$, (D) aspecto del bosque luego de un incendio de severidad media (Parque Nacional Tolhuaca).

(A) Diameter structure of Araucaria-Nothofagus old-growth forest killed by fire (2002), (B) appearance of the forest after the high-severity fire, (C) Diameter structure of Araucaria-Nothofagus old-growth forest including trees without canopy damage (VSD), trees with canopy damage (VCD), and trees killed $(\mathrm{M})$ by fire $(\mathrm{A}=$ Araucaria $; \mathrm{N}=$ Nothofagus $)$, (D) Appearance of the forest after the mid-severity fire. 
TABLA 2

Frecuencia (\% ocurrencia) y cobertura media relativa $(\%)$ de especies durante la tercera temporada de crecimiento luego de un incendio en el Parque Nacional Tolhuaca. Se incluye rodales afectados con una severidad media (cercano a 20-60\% individuos arbóreos vivos $>5 \mathrm{~cm}$ Dap) y alta $(<20 \%$ individuos arbóreos vivos de $>5 \mathrm{~cm}$ Dap) (M.E. González, resultados no publicados)

Frequency (\% occurrence) and mean cover (\%) of the species in the third growing season after fire in Tolhuaca National Park. It includes stands affected with a mid-severity fire (ca. 20-60\% of trees alive $>5 \mathrm{~cm} \mathrm{Dbh}$ ) and high-severity fire (< $20 \%$ of trees alive $>5 \mathrm{~cm} \mathrm{Dbh})$

\begin{tabular}{|c|c|c|c|c|}
\hline \multirow[t]{2}{*}{ Especies } & \multicolumn{2}{|c|}{$\begin{array}{c}\text { Media } \\
(\mathrm{n}=90)\end{array}$} & \multicolumn{2}{|c|}{$\begin{array}{c}\text { Alta } \\
(\mathrm{n}=90)\end{array}$} \\
\hline & FR (\%) & $\mathrm{CR}(\%)$ & $\overline{\mathrm{FR}(\%)}$ & CR $(\%)$ \\
\hline \multicolumn{5}{|l|}{ Árboles $(<2 \mathrm{~m})$} \\
\hline Araucaria araucana (Mol.) K. Koch & 2,2 & 0,65 & 1,8 & 0,19 \\
\hline Nothofagus dombeyi (Mirb.) Oerst. & 3,5 & 0,82 & 0,3 & 0,01 \\
\hline Nothofagus pumilio (Poepp. et Endl.) & 4,0 & 2,7 & & \\
\hline Nothofagus nervosa Phil. & 1,5 & 1,3 & 0,3 & 0,9 \\
\hline Embothrium coccineum J.R. et G. Forster & 3,0 & 2,8 & 3,0 & 3,6 \\
\hline Lomatia hirsuta Lam. Diels ex Macbr. & 0,3 & 0,9 & & \\
\hline \multicolumn{5}{|l|}{ Arbustos } \\
\hline Chusquea culeou Desv. & 14,4 & 36,3 & 9,8 & 20,3 \\
\hline Ovidia andina (OPEP. Et. Ende.) Meissn. & 0,2 & 0,52 & & \\
\hline Maytenus disticha (Hook.f.) Urban & 8,7 & 6,8 & 1,5 & 0,18 \\
\hline Ribes magellanicum Poir. & 2,7 & 1,5 & 5,7 & 8,0 \\
\hline Baccharis spp. & 2,7 & 2,5 & 1,2 & 0,51 \\
\hline Senecio spp.(1) & 0,2 & 0,21 & & \\
\hline Berberis spp. & 0,2 & 0,21 & 0,6 & 0,9 \\
\hline Myrceugenia spp. & 2,2 & 3,0 & 2,7 & 0,87 \\
\hline Drimys andina (Reiche)R.A.Rodr. et Quez. & 0,7 & 1,47 & 1,5 & 2,64 \\
\hline Pernettya spp. & 3,5 & 3,74 & 5,1 & 2,34 \\
\hline Azara lanceolata Hook. f. & 0,5 & 1,23 & 1,5 & 0,87 \\
\hline Myochilos oblonga R. et. P. & 1,7 & 1,32 & 0,6 & 0,08 \\
\hline \multicolumn{5}{|l|}{ Trepadoras, helechos, briófitas } \\
\hline Dioscorea brachybotrya OPEP. & 5,7 & 1,7 & 8,3 & 3,64 \\
\hline Muehlenbeckia hastulata (J.E. Sm.) Johnst. & 5,4 & 6,13 & & \\
\hline Blechnum penna-marina (Poiret Kuhn) & 0,2 & 0,21 & 0,6 & 0,43 \\
\hline Musgo & 5,5 & 7,5 & 3,6 & 7,52 \\
\hline Marchantia spp. & 3,5 & 3,7 & 1,5 & 3,18 \\
\hline \multicolumn{5}{|l|}{ Hierbas } \\
\hline Senecio spp.(2) & 1,2 & 0,24 & 0,9 & 0,04 \\
\hline Senecio spp. (3) & 5,0 & 2,91 & 14 & 18,24 \\
\hline Hypochoeris radicata $\mathrm{L}$. & 0,7 & 0,06 & 3,6 & 0,32 \\
\hline Lathyrus spp. & 5,1 & 1,02 & & \\
\hline Acaena spp. & 0,9 & 0,78 & & \\
\hline Solanum spp. & 0,6 & 0,02 & & \\
\hline Adesmia spp. & 1,8 & 0,37 & & \\
\hline Alstroemeria aurantiaca D. Don & 4,5 & 3,8 & 4,8 & 5,83 \\
\hline Libertia spp. & 2,0 & 0,43 & 3,9 & 2,13 \\
\hline Graminea & 0,2 & 0,87 & 1,5 & 2,58 \\
\hline Cirsium vulgare (Savi) Ten. & 0,2 & 0,01 & 1,8 & 1,0 \\
\hline Taraxacum officinale Weber & 0,2 & 0,04 & 0,3 & 0,07 \\
\hline Festuca spp. & 0,2 & 0,52 & 4,8 & 3,18 \\
\hline Perezia spp. & 2,5 & 1,70 & 0,3 & 0,89 \\
\hline Rubus geoides J.E. Sm. & 1,7 & 0,7 & & \\
\hline Galium spp. & 1,2 & 0,68 & & \\
\hline Juncus spp. & 0,2 & 0,21 & & \\
\hline Quinchamalium dombeyi Brongn. & 0,2 & 0,04 & & \\
\hline Viola reichei Skottsb. & 3,0 & 0,8 & & \\
\hline Adenocaulon chilense Less. & 3,5 & 3,25 & & \\
\hline Ranunculus spp. & 0,2 & 0,04 & & \\
\hline Lycopodium spp. & 3,2 & 2,5 & & \\
\hline Especie 1 & 0,2 & 0,04 & & \\
\hline Especie 2 & 0,3 & 0,07 & & \\
\hline Especie 3 & 0,3 & 0,01 & & \\
\hline
\end{tabular}

FR = frecuencia relativa; $C R=$ cobertura media relativa. La nomenclatura sigue a Marticorena \& Quezada (1985) 
Las actividades de madereo de salvamento en áreas recientemente afectadas por disturbios menoscaban fuertemente su potencial de recuperación. Si bien los bosques afectados por incendios (e.g., Nothofagus spp.) son generalmente capaces de establecer en un lapso relativamente rápido una densa regeneración, el periodo postfuego representa una etapa en que ellos se encuentran en un particular estado de vulnerabilidad. Ciertamente, la vegetación en una etapa temprana postfuego es de menos resiliencia que la de un bosque en una etapa de desarrollo más avanzada, y por lo tanto los efectos interactivos y combinados de alteraciones consecutivas (e.g., fuego seguido de madereo de salvamento) pueden afectar más críticamente taxa no adaptados y otros procesos ecológicos (Paine et al. 1998, Lindenmayer et al. 2004). Numerosos ejemplos están a la vista en que la combinación consecutiva de disturbios donde generalmente uno de ellos fue de origen antrópico- ha llevado al fracaso o muy lenta recuperación de estos ecosistemas. Por ejemplo, los grandes incendios de 1940 en la Región de Aysén, que fueron seguidos por una intensa presión ganadera resultaron en la eliminación de la cobertura arbórea de miles de hectáreas (Sierra 1978, Donoso 1983). Ejemplos similares pueden ser mencionados para lo ocurrido con los bosques de Alerce (Fitzroya cupressoides), Ciprés (Pilgerodendron uviferum) y Araucaria, en que el fuego, madereo (pre o postincendio), y ganadería causó ya sea la eliminación o cambio del tipo de vegetación o dirigió estos ecosistemas a un lento y crítico proceso de recuperación que puede ser visto hasta el presente (González 2002, Bannister 2004, Soto 2004, Torres 2004).

A nivel mundial existen numerosas experiencias que revelan cómo el impacto de actividades de madereo en áreas recientemente afectadas por disturbios menoscaban fuertemente el potencial de recuperación de estas mismas. Por ejemplo, el extensivo madereo realizado luego del huracán de 1938 que afectó los bosques del noreste de los Estados Unidos tuvo por resultado enormes efectos en la hidrología y muchos otros procesos ecosistémicos (Foster et al. 1997). En el sudeste asiático, luego de los incendios ocurridos en el 1997-98 que afectaron cerca de 10 millones de hectáreas de bosques tropicales fueron madereados a gran escala generando un fuerte menoscabo en el potencial regenerativo de los bosques (Van Nieuwstadt et al. 2001). Similares impactos negativos han sido documentados para bosques en el sudeste de Australia (Lindenmayer 1996).

$\mathrm{Si}$ bien los bosques dominados por Araucaria-Nothofagus responden y pueden recuperarse natural y favorablemente después de incendios, el efecto de disturbios sucesivos, como el salvamento vía madereo de árboles muertos, puede impactar negativamente esa potencialidad de recuperación. A continuación se resumen las principales experiencias y efectos en relación al impacto del madereo de rescate: (i) impacto del madereo de salvamento en áreas de alta sensibilidad. En bosques que habitan comúnmente áreas de una topografía quebrada con suelos frágiles (e.g., Araucaria) son altamente sensibles a procesos erosivos. El madereo especialmente con maquinaria pesada convencional (skidder y tractores) acelera la erosión del suelo y aumenta su compactación lo que tiene un efecto directo en la tasa de sedimentación y escorrentía superficial, y consecuentemente en el ciclo hidrológico, sistemas acuáticos e integridad biológica del suelo (McIver \& Starr 2000). También, si existe regeneración de avanzada postincendio, el madereo puede tener un fuerte efecto sobre su desarrollo y supervivencia. (ii) La construcción de caminos de acceso tiene variados impactos ecológicos negativos en áreas quemadas. En general, la construcción de nuevos caminos de accesibilidad a las áreas afectadas genera una serie de impactos negativos en el ecosistema acuático, incluyendo la disrupción de la hidrología y el aumento agudo de la sedimentación (Forman 2000, Jones et al. 2000). Estos procesos pueden constituirse en un problema crítico para peces y otras especies acuáticas sensibles (Baxter et al. 1999). Además, nuevos caminos que permiten el acceso a las áreas quemadas promueven el arribo de especies alóctonas no deseadas que pueden intervenir seriamente con el proceso sucesional y de recuperación natural (Beschta et al. 1995, Trombulack \& Frissell 2000). Por otro lado, barreras como caminos pueden limitar o impedir procesos de dispersión de organismos como invertebrados, y ciertos mamíferos y plantas (Baur \& Baur 1990, Burnett 1992). Por último, la construcción de caminos puede resultar en un aumento de la 
ignición humana ya sea accidental o intencional con consecuencias muy negativas para la conservación de estos ecosistemas (Franklin \& Forman 1987). (iii) El legado biológico postdisturbio es de crítica importancia en la mantención de la diversidad biológica y de recuperación de la comunidad. El legado biológico entendido como árboles vivos y muertos, y otros organismos, y estructuras químicas y físicas retenidas luego del disturbio actúan como refugios que permiten a algunas especies sobrevivir y subsecuentemente recolonizar sitios postdisturbio, así como también atraer la inmigración de otros organismos (Franklin et al. 2000). De esta forma el legado biológico tiene una importante influencia en la trayectoria sucesional del ecosistema. El madereo de aprovechamiento, debido a que implica la remoción directa del componente arbóreo, disminuye la densidad de la mayoría de las aves que nidifican en cavidades y también la capacidad de recolonización de comunidades únicas de epifitas, artrópodos y vertebrados asociados a grandes y viejos individuos arbóreos (Hunter 1995, Saab \& Dudley 1998). Conjuntamente, el madereo puede afectar los procesos de regeneración derivado tanto del daño mecánico directo -a la nueva regeneración y la de avanzada- como de los cambios en el microclima. El legado de árboles vivos y muertos (snags) puede mitigar el estrés medioambiental asociado a factores como el viento, radiación solar directa, desecación y extremos de temperatura (Franklin \& Armesto 1996). El rol de los árboles como sitios de anidación, alimentación o paradero de aves (Willson et al. 1994) pueden contribuir efectivamente a la dispersión de propágulos y colonización de sitios perturbados (Franklin \& Armesto 1996). Además, troncos en el piso no solo reducen la erosión actuando como barreras al movimiento del suelo sino constituyen un centro de actividad biológica que incluye hongos micorrícicos, bacterias fijadoras de nitrógeno, anfibios, y pequeños mamíferos (Franklin et al. 1985, Harmon et al. 1986).

\section{CONSIDERACIONES FINALES}

El fuego ha sido un agente natural crucial en la dinámica de los ecosistemas boscosos templados de Chile y Argentina. Los grandes incendios ocurridos recientemente en bosques andinos de Araucaria araucana, causados por condiciones climáticas -relativamente infrecuentes - generadoras de tormentas eléctricas, señalan la naturalidad del fuego en estos bosques milenarios. En estos ecosistemas, el clima y el ser humano han influido significativamente en la frecuencia y magnitud de los incendios. Estudios dendroecológicos han confirmado la estrecha relación entre la variabilidad climática interanual y la ocurrencia de grandes incendios durante los últimos mil años (Kitzberger \& Veblen 1997, Veblen et al. 1999, González \& Veblen 2006). Además, el uso de estos espacios por comunidades indígenas, y posteriormente por colonos eurochilenos, tuvo un impacto importante en la ocurrencia de incendios. Así, estos cambios inducidos por el ser humano sustentan la idea que el régimen de incendios y los patrones de vegetación (mosaico de edad de rodales en el paisaje) estarían fuera o alejados de sus rangos naturales o históricos de variación (González 2005). El régimen de fuego natural estimado estaría caracterizado en esencia por una menor frecuencia (respecto de los últimos 150 años) de incendios tanto catastróficos y superficiales, y un mosaico de bosques en distintos estados de desarrollo. Sin embargo, posterior a la colonización de la Araucanía estos ecosistemas han experimentado una mayor frecuencia de incendios, tanto de baja y alta severidad, lo que ha resultado en un paisaje fisonómicamente distinto, más fragmentado, y actualmente dominado por poblaciones postfuego de Nothofagus de 50-150 años.

La consideración de algunos factores económicos y culturales conduce a ser precavidos al momento de implementar proyectos de salvamento de maderas muertas en estos bosques. La revisión histórica de las prácticas ocurridas con la especie Alerce (Fitzroya cuppressoides), en que solo la corta de árboles muertos está permitida, revelan los efectos negativos que estas prácticas pueden tener en la vegetación remanente, y además en el potencial "incentivo" (perverso) a la quema intencional de estos bosques. En el caso particular de Araucaria y coincidente con la reapertura temporal de su explotación en 1988, no solo la conservación de la especie fue amenazada, sino que también esta situación 
derivó en un litigio por los derechos de la tierra entre la comunidad Pehuenche de Quinquén y propietarios privados, lo cual finalmente resultó en el desembolso de varios millones de dólares (U\$ 6 millones 150 mil) por parte del Estado de Chile para el asentamiento definitivo de la comunidad en el valle de Quinquén (Lara et al. 1996).

La proposición de distintos proyectos y actividades a ejecutar en las áreas recientemente quemadas, tanto privadas como públicas, deben ser evaluados cautelosamente a la luz de los actuales conocimientos. De las experiencias y consideraciones previas claramente se desprende que la intervención humana postfuego puede sustancialmente retrasar el proceso de recuperación, remover elementos clave para este, o acentuar el daño. En muchas situaciones específicas las consecuencias adversas son difíciles o imposibles de predecir. Por otro lado, los incendios que afectaron a los bosques de Araucaria representan una excelente oportunidad para incrementar nuestro conocimiento respecto del rol y efecto del fuego en la diversidad y dinámica de estos ecosistemas forestales. De suma importancia resulta considerar que actualmente en Chile solo existe un $50 \%$ (cerca de 250.000 ha) de las poblaciones originales de Araucaria (Lara et al. 1999), de las cuales un gran porcentaje ha sido directa o indirectamente impactada por actividades humanas durante el siglo pasado. Así, tomando en cuenta sus problemas de conservación y relevancia científica y cultural para Chile y sus pueblos originarios, las agencias y sus políticas de manejo deberían incentivar una acción fuertemente orientada a comprender estos procesos ecológicos de manera de promover adecuadas medidas de mitigación y restauración. A partir de las primeras evaluaciones realizadas en el Parque Nacional Tolhuaca, estos ecosistemas muestran una habilidad natural para recuperarse espontáneamente luego de incendios (M.E. González, resultados no publicados), y por lo tanto, una restauración de tipo pasiva podría ser implementada. Sin embargo, la presión ganadera que actualmente ocurre en muchas áreas quemadas -incluido Tolhuaca- podría estar poniendo en riesgo esta recuperación natural y menos costosa del ecosistema.

\section{AGRADECIMIENTOS}

En sus diferentes etapas este trabajo ha recibido el apoyo de las siguientes instituciones: Fundación Andes (C-13860), Internacional Foundation for Science (IFS, D/3124-2), y Dirección de Investigación y Desarrollo de la Universidad Austral de Chile (DID S-2005-08). Reciba nuestro agradecimiento la Corporación Nacional Forestal (CONAF) por las facilidades y permisos otorgados para la realización del trabajo de colecta de material en Parques y Reservas Nacionales. Se agradece la colaboración en el trabajo de terreno y laboratorio de los estudiantes Juan Quezada y Paul Szejner. Este artículo fue mejorado gracias a los comentarios y sugerencias de dos revisores anónimos, y a los del editor asociado J.J. Armesto.

\section{LITERATURA CITADA}

BAQUEDANO RE (1914) Estudio sobre la araucaria chilena (Araucaria imbricata). Sección Impresiones del Instituto Meteorológico, Santiago, Chile. 29 pp.

BANNISTER J (2004) Estado de conservación de Pilgerodendron uviferum (D. Don) Florin en el área norte de la cordillera de Pirulil. Tesis de pregrado, Facultad de Ciencias Forestales, Universidad Austral de Chile, Valdivia, Chile. 49 pp.

BAUR A \& B BAUR (1990) Are roads barriers to dispersal in the land snail Arianta arbustorum? Canadian Journal of Zoology 68: 613-617.

BAXTER CV, CA FRISSELL \& FR HAUER (1999) Geomorphology, logging roads, and the distribution of bull trout (Salvelinus confluentus) spawning in a forested river basin: Implications for management and conservation. Transactions of the North American Fisheries Society 128:854-867.

BEKESSY SA, A LARA, ME GONZÁLEZ, M CORTÉS, L GALLO, AC PREMOLI \& AC NEWTON (2004) Variación en Araucaria araucana (Molina) K. Koch (Araucaria o Pehuén). En: Donoso C, A Premoli, L Gallo \& R Ipinza (eds) Variación intraespecífica en las especies arboreas de los bosques templados de Chile y Argentina: 215-231. Editorial Universitaria, Santiago, Chile.

BESCHTA RL, CA FRISSELL, R GRESSWELL, R HAUER, JR KARR, GW MINSHALL, DA PERRY \& JJ RHODES (1995) Wildfire and salvage logging: recommendations for ecologically sound post-fire salvage logging and other post-fire treatments on federal lands in the west. Report, Corvallis, Oregon, USA. 9 pp.

BURNETT S (1992) Effects of a rainforest road on movements of small mammals: mechanisms and implications. Wildlife Research 19: 95-104.

CONAF (2002) Antecedentes sobre impacto de incendios forestales en la IX Región. Documento de visita a la zona afectada por Incendios, Temuco, Chile. 16 pp.

DONOSO C (1983) Modificaciones del paisaje chileno a lo largo de la historia. Simposio Desarrollo y 
Perspectivas de las disciplinas forestales en la Universidad Austral de Chile, Facultad de Ciencias Forestales, Universidad Austral de Chile, Valdivia, Chile.

DONOSO C \& A LARA (1996) Utilización de los bosques nativos en Chile: pasado, presente y futuro. En: Armesto JJ, C Villagrán \& MK Arroyo (eds) Ecología de los bosques nativos de Chile: 363-387. Editorial Universitaria, Santiago, Chile.

ECHEVERRÍA C (2002) Ecological disaster in southern Chile. Plant Talk (USA) 28: 14.

ELIZALDE R (1958) La sobrevivencia de Chile. Departamento de Conservación y Administración de Recursos Agrícolas y Forestales, Ministerio de Agricultura, Santiago, Chile. 172 pp.

FORMAN RT (2000) Estimate of the area affected ecologically by the road system in the United States. Conservation Biology 14:31-35.

FOSTER DR, JD ABER, JM MELILLO, RD BOWDEN \& FA BAZZAZ (1997) Temperate forest response to natural catastrophic disturbance and chronic anthropogenic stress: rethinking the 1938 Hurricane and the impact of physical disturbance vs. chemical and climate stress on forest ecosystems. BioScience 47: 437-445.

FRANKLIN JF \& RT FORMAN (1987) Creating landscape patterns by forest cutting: ecological consequences and principles. Landscape Ecology 1: 5-18.

FRANKLIN J \& JJ ARMESTO (1996) Una alternativa de manejo para los bosques nativos chilenos. Ambiente y Desarrollo (Chile) 12: 69-79.

FRANKLIN JF, JA MACMAHON, FJ SWANSON \& JR SEDELL (1985) Ecosystem responses to the eruption of Mount St. Helens. National Geographic Research 1 (Spring): 196-215.

FRANKLIN JF, D LINDENMAYER, JM MACMAHON, A MCKEE, J MAGNUSSON, DA PERRY, R WAIDE \& D FOSTER (2000) Threads of continuity: ecosystem disturbances, biological legacies and ecosystem recovery. Conservation Biology in Practice (USA) 1: 8-16.

GONZÁLEZ ME (2002) Fire history of AraucariaNothofagus forests in the Andean cordillera of south-central Chile. Dissertation, University of Colorado, Boulder, Colorado, USA. 158 pp.

GONZÁLEZ ME (2005) Fire history data as reference information in ecological restoration. Dendrochrologia (Germany) 22: 149-154.

GONZÁLEZ ME, TT VEBLEN \& JS SIBOLD (2005) Fire history of Araucaria-Nothofagus forest in Villarrica National Park, Chile. Journal of Biogeography 32: 1187-1202.

GONZÁLEZ ME \& TT VEBLEN (2006) Climatic influences on fire in Araucaria araucanaNothofagus forests in the Andean cordillera of south-central Chile. Ecoscience 13: 342-350.

GLENN-LEWIN DC, RK PEET \& TT VEBLEN (eds) (1992) Plant succession theory and prediction. Chapman \& Hall, New York, New York, USA. 352 pp.

HARMON ME, JF FRANKLIN, FJ SWANSON, P SOLLINS, SV GREGORY, JD LATTIN, NH ANDERSON, SP CLINE, NG AUMEN, JR SEDELL, GW LIENKAEMPER, K CROMACK \& KW CUMMINS (1986) Ecology of coarse woody debris in temperate ecosystems. Advances in Ecological Research 15: 133-302.

HEUSSER CJ (1983) Quaternary pollen record from Laguna de Tagua Tagua, Chile. Science 219: 14291432 .
HEUSSER CJ (1994) Paleoindians and fire during the late Quaternary in southern South America. Revista Chilena de Historia Natural 67: 435-442.

HUNTER M (1995) Residual trees as biological legacies. Cascade Center for Ecosystem Management, Communique 2, Department of Forest Science, Oregon State University and United States Department of Agriculture, Forest Service, Corvallis, Oregon (USA). 27 pp

MCIVER JD \& L STARR (2000) Environmental effects of postfire logging: literature review and annotated bibliography. General Technical Report PNWGTR-486. Pacific Northwest Research Station, Forest Service, United Stated Department of Agriculture. $72 \mathrm{pp}$

JONES JA, FJ SWANSON, BC WEMPLE \& KU SNYDER (2000) Effects of roads on hydrology, geomorphology, and disturbance patches in stream networks. Conservation Biology 14: 76-85.

KITZBERGER T \& TT VEBLEN (1997) Influences of humans and ENSO on fire history of Austrocedrus chilensis woodlands in northern Patagonia, Argentina. Ecoscience 4: 508-520.

KITZBERGER T, TT VEBLEN \& R VILLALBA (1997) Climatic influences on fire regimes along a rain forest-to-xeric woodland gradient in northern Patagonia, Argentina. Journal of Biogeography 24: $35-47$.

LARA A, C DONOSO \& ARAVENA JC (1996) La conservación de los bosques nativos de Chile: problemas y desafíos. En: Armesto JJ, C Villagrán \& MK Arroyo (eds) Ecología de los bosques nativos de Chile: 335-362. Editorial Universitaria, Santiago, Chile.

LARA A, ME SOLARI, P RUTHERFORD, O THIERS, R TRECAMAN, R MOLINA, R PRIETO y C MONTORY (1999). Cobertura de la vegetación original de la ecoregión de los bosques valdivianos en Chile hacia 1550. Informe Técnico, Proyecto FB 49 World Wildlife Found/Universidad Austral de Chile, Valdivia, Chile

LINDENMAYER DB (1996) Wildlife and woodchips: Leadbeater's possum as a test case of sustainable forestry. University of New South Wales Press, Sidney, Australia. $156 \mathrm{pp}$.

LINDENMAYER DB \& JF FRANKLIN (2002) Conserving forest biodiversity: a comprehensive multiscaled approach. Island Press, Washington, District of Columbia, USA. $351 \mathrm{pp}$.

LINDENMAYER DB, DR FOSTER, JF FRANKLIN, ML HUNTER, RF NOSS, FA SCHMIEGELOW \& D PERRY (2004) Salvage harvesting policies after natural disturbance. Science 303: 130.

MARTICORENA C \& M QUEZADA (1985) Catálogo de la flora vascular de Chile. Gayana Botánica (Chile) 42: $5-155$

MONTALDO P (1974) La bio-ecología de Araucaria araucana (Mol.) Koch. Boletín Instituto Forestal Latino-Americano de Investigación y Capacitación 46/48: 3-55

MONTECINOS A \& P ACEITUNO (2003) Seasonality of the ENSO-related rainfall variability in central Chile and associated circulation anomalies. Journal of Climate 16: 281-296.

PAINE RT, TEGNER MJ \& EA JOHNSON (1998) Compounded perturbations yield ecological surprises. Ecosystems 1: 535-545.

PEÑALOZA R (2006) Zonificación de la severidad de un incendio natural y su descripción topográfica cuantitativa en el Parque Nacional Tolhuaca, 
Novena Región, Chile. Tesis de pregrado, Facultad de Ciencias Forestales. Universidad Austral de Chile, Valdivia, Chile. 36 pp.

PEREIRA E (1965) El desenvolvimiento histórico-étnico de la población. Geografía Económica de Chile, Editorial Universitaria, Santiago, Chile.

PICKETT STA \& PS WHITE (eds) (1985) The ecology of natural disturbance and patch dynamics. Academic Press, San Diego, California, USA. 472 pp.

SAAB V A \& JG DUDLEY (1998). Responses of cavitynesting birds to stand-replacement fire and salvage logging in ponderosa pine/Douglas-fir forests of southwestern Idaho. United States Department of Agriculture, Forest Service, Rocky Mountain Research Station, Research Paper, RMRS-RP-11.

SIERRA V (1978) Erosión, un mal que corroe las tierras de Aisén. Trapananda (Chile) 1: 97-101.

SOTO D (2004) Estado de conservación de Pilgerodendron uviferum (D. Don) Florin en la cordillera de la costa, Valdivia. Tesis de pregrado, Facultad de Ciencias Forestales, Valdivia, Chile. 35 pp.

SPIES T \& M TURNER (1999) Dynamic forest mosaics. En: Hunter ML (ed) Maintaining biodiversity in forest ecosystems: 95-160. Cambridge University Press, New York, New Cork, USA.

TORRES G (2004) Efecto de incendios sobre el patrón de regeneración arbórea en formaciones de alerce (Fitzroya cuppressoides) de la cordillera de Yerbas Buenas, Décima Región de Los Lagos, Chile. Tesis de Magíster en Ciencias, Universidad de Los Lagos, Osorno, Chile. 90 pp.
TROMBULAK SC \& CA FRISSELL (2000) Review of ecological effects of roads on terrestrial and aquatic communities. Conservation Biology 14: 18-30.

TURNER MG, WL BAKER, CJ PETERSON \& RK PEET (1998) Factors influencing succession: lessons from large, infrequent natural disturbances. Ecosystems 1: $511-523$

VAN NIEUWSTADT MGL, D SHEIL \& $\mathrm{K}$ KARTAWINATA (2001) The ecological consequences of logging in the burned forests of east Kalimantan, Indonesia. Conservation Biology 15: 1183-1186.

VEBLEN TT, BR BURNS, T KITZBERGER, A LARA \& R VILLALBA (1995) The ecology of the conifer of Southern South America. In: Enright NJ, RS Hill (eds) Ecology of the Southern Conifers: 120-155. Melbourne University Press, Victoria, Australia.

VEBLEN TT, C DONOSO, T KITZBERGER \& AJ REBERTUS (1996) Ecology of southern Chilean and Argentinean Nothofagus forests. En: Veblen TT, RS Hill \& J Read (eds) The ecology and biogeography of Nothofagus forests: 293-353. Yale University Press, New Haven, Connecticut, USA.

VEBLEN TT, T KITZBERGER, R VILLALBA \& J DONNEGAN (1999) Fire history in northern Patagonia: the roles of humans and climatic variation. Ecological Monographs 69: 47-67.

WILLSON MF, TL DE SAMTO, C SABAG \& JJ ARMESTO (1994) Avian communities of fragmented south-temperate rainforests in Chile. Conservation Biology 8: 508-520. 
